\title{
STRUCTURAL CHARACTERIZATION OF CO-DOPED ZnO NANOPARTICLES USING X-RAY DIFFRACTION AND RAMAN SPECTROSCOPY
}

\author{
Castro, T. J. ${ }^{1}{ }^{*}$; da Silva, S. W. ${ }^{1}$; Nakagomi, F. ${ }^{1}$; Franco Júnior, A. ${ }^{2}$; Pessoni, H. V. \\ S. ${ }^{2}$; Morais, P. C. ${ }^{1}$ \\ ${ }^{1}$ Instituto de Física, Universidade de Brasília, Brasília, Distrito Federal, Brazil \\ 2 Instituto de Física, Universidade Federal de Goiás, Goiânia, Goiás, Brasil.
}

*tiago.castroifb.edu.br

Zinc Oxide $(\mathrm{ZnO})$ has received a great deal of attention due the wide range of its technological applications, including transparent conducting electrodes for solar cells, transparent ultraviolet protection films, low-voltage and short-wavelength electro-optical devices. In addition, when doped with transition metal atoms (TM), ZnO exhibits ferromagnetic and semiconducting properties, forming a diluted magnetic semiconductor (DMS). This type of material has been extensively studied due to its potential use in spintronic devices as a consequence of its predicted room temperature ferromagnetism (RTFM). In this work, we have performed the structural characterization of Co-doped $\mathrm{ZnO}$ nanoparticles with different cobalt contents. The combustion reaction method was chosen for samples preparation and their properties were investigated by X-Ray Diffraction (XRD) and Raman Spectroscopy. The diffraction patterns and Raman spectra of all samples indicated the formation of $\mathrm{ZnO}$ hexagonal wurtzite phase (space group $\left.\mathrm{P}_{3} m c\right)$. Results from XRD and Raman spectroscopy exhibited evidences of continuous changes in the structural parameters $(a, c$ and $u)$ of $\mathrm{ZnO}$ with increasing dopant content. Samples with higher Co content also revealed the existence of an additional impurity phase $\left(\mathrm{Co}_{3} \mathrm{O}_{4}\right)$. Rietveld refinement method (performed with EXPGUI-GSAS software) was employed to study the collected XRD data. By using the Stephens Model, it was possible to verify the occurance of anisotropic strain along different axes. However, only the effects of compressive $\operatorname{strain}\left(\varepsilon_{c c}<0\right)$ was not sufficient to explain the variation of the Raman shift of the $E_{2}^{\text {high }}$ mode. To investigate this discrepancy, the Phonon Confinement Model was employed, which showed that the correlation length $L$ (spatial region in which the phonon is confined) also decreases as Co content increases. 